\section{Rational Investigations in Irritable Bowel Syndrome}

Irritable Bowel Syndrome is a functional gastrointestinal disorder with an estimated prevalence of around $10 \%$. The approach to investigation is variable and influenced by the symptom severity, clinician experience and the clinical setting but based around the principle of careful clinical assessment, early diagnosis and limiting to necessary and relevant tests. In this issue Christopher Black and Alexander Ford summarise the evidence base and provide practical guidance. Diagnostic criteria should be present as per the Rome Criteria. Routine bloods are acceptable but have a low diagnostic yield. Coeliac testing is reasonable. Faecal Calprotectin should be considered, especially if there is diarrhoea, proceeding to colonoscopy if the test is positive. More intensive investigation should be reserved for specific cases and dependant on the history, examination and red flags if present. Colonoscopy should be considered in any patient with alarm features for colorectal cancer or if microscopic colitis is suspected (even if the calprotectin is normal). If bile acid diarrhoea is suspected then 23-seslo-25-homotaurocholic acid (SeHCAT) scanning should be considered. Breath testing and testing for small intestinal bacterial overgrowth are not helpful and a trial of treatment probably the more effective test. In summary therefore an early positive diagnosis of IBS and limiting the need for clinical investigation is advocated which creates the opportunity for initiation of earlier symptom-specific treatment. (See page 140 and listen to the accompanying podcast)

\section{Nutrition in liver disease}

Key to the management of liver disease - from compensated cirrhosis through to liver failure - is the early recognition and treatment of malnutrition particularly when associated with Sarcopenia (reduction in muscle, function and strength). Malnutrition is generally associated with under nutrition although does include 'over' nutrition as seen in Non Alcoholic Fatty Liver Disease when Sarcopenia can also be present and the term 'Sarcopenic Obesity' is increasingly used. In this issue Dhaliwal and colleagues use a case based approach and evidence based guidance to discuss the nutritional management of compensated cirrhosis and decompensated cirrhosis during the acute and chronic phase. Key messages include the protection of muscle mass, minimising fasting (and catabolism), protein targets and functional assessment/intervention with the strategy being to 'use it or lose it'. (See page 155 and listen to the accompanying podcast)

\section{Ocular manifestations of gastrointestinal disease}

Important and can present as an emergency. In a curriculum based review this month Imam and Haboubi discuss ocular manifestations of gastrointestinal disease including epidemiology, detailed description of the clinical features including useful images, treatment and complications. In Inflammatory Bowel Disease this includes episcleritis, scleritis, uveitis and cataract (secondary to steroids). Episcleritis (the most common) mirrors disease activity and improves with disease control. Less common are scleritis (including posterior sclera which can result in visual loss and may be complicated by retinal detachment or optic neuritis) and uveitis which require prompt recognition and treatment. Other conditions discussed include Keratoconjunctivitis Sicca (dry eye), Optic Disc Drusen (Alagille Syndrome), Kayser Fleischer rings (Wilson's Disease), Xanthelasma (primary biliary cirrhosis, chronic liver disease, hyperlipidaema). The authors also discuss ophthalmological manifestations of vitamin A deficiency including xerophthalmia, night blindness, Bitot's spots, keratitis and keratomalacia. There are multiple gastrointestinal causes of vitamin A deficiency and treatment is with vitamin A and treatment of the underlying cause although prognosis is variable. The article is essential reading for trainees and a useful and practical update for clinicians in practice. (See page 162)

\section{Development and implementation of a commissioned pathway for the identification and stratification of liver disease in the community}

The need to detect liver disease early to allow intervention and potentially change the course of the disease is uncontroversial.
In this issue Chalmers and colleagues describe the development and implementation of the Nottingham liver disease stratification pathway. Patients were referred to the pathway with a raised AST/ALT ratio, harmful alcohol use or risk or presence of non-alcoholic fatty liver disease. Intervention was transient elastography (TE) to look at liver stiffness and brief lifestyle intervention. This was in primary care with advice on interpretation and referral guidance (figure 1 in the paper). 222 of 968 patients referred had raised TE with 57 having liver stiffness indicative of advanced chronic liver disease, some of whom would have been missed using standard referral criteria. The pathway proved feasible and effective with the potential to improve early detection rates and stratify referrals for specialist input. The model could easily be adopted in other settings. There is an excellent accompanying commentary - On the path to developing significant liver disease. (See pages 84 and 86)

\section{Paediatric parenteral nutrition - current issues}

Parental Nutrition (PN) has transformed the prognosis of infants and children with intestinal failure with significant advances in the field over the last 10-15 years. In this issue Elena Cernat and John Puntis discussed the principles and practice and summarise recent guidance from the European Society of Paediatric Gastroenterology, Hepatology and Nutrition (ESPGHAN) and the European Society for Clinical Nutrition and Metabolism (ESPEN). There are useful sections PN components - energy, amino acids, lipid, carbohydrate, fluid and electrolytes, micronutrients and vitamins. This includes energy requirements and when to start PN (can potentially delay for up to a week in critically ill children), avoidance of soybean oil emulsions and use of composite emulsions with or without fish oil if $\mathrm{PN}$ is required for more a few days and the use of Taurolodine line locks to reduce catheter related sepsis in children on long term PN. The guidance is useful, practical and up to date. (See page 148)

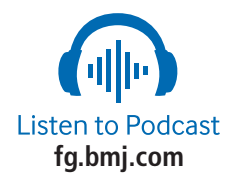

